Discussion. Accurate reduction of a long bone fracture is important, since it has been shown that degenerative changes can follow even minimal malalignment (Kettelkamp et al 1988). The maximum acceptable angular deformity in the tibia or femur has not been accurately determined (Ting et al 1987), but $15^{\circ}$ of femoral and $5^{\circ}$ of tibial angulation have been accepted (Mooney and Claudi 1984). Values greater than this may lead to deterioration of the articular surfaces.

The method described here is simple, reproducible, does not affect the surgical field, is more accurate than conventional plain radiography, involves less radiation to the patient and theatre staff and saves theatre time.

One or more of the authors have received or will receive benefits for personal or professional use from a commercial party related directly or indirectly to the subject of this article. In addition benefits have also been or will be directed to a research fund, foundation, educational institution, or other non-profit institution with which one or more of the authors is associated.

\section{REFERENCES}

Kettelkamp DB, Hillberry BM, Murrish DE, Heck DA. Degenerative arthritis of the knee secondary to fracture malunion. Clin Orthop $1988 ; 234: 159-69$.

Mooney V, Clandi BF. Fractures of the shaft of the femur. In : Rockwood CA Jr and Green DP, eds. Fractures in adults. Vol 2. 2nd ed. Philadelphia, etc: JB Lippincott Co, 1984:1357-1428.

Moreland JR, Bassett LW, Hanker GJ. Radiographic analysis of the axial alignment of the lower extremity. J Bone Joint Surg [Am] 1987; 69-A :745-9.

Ting AJ, Tarr RR, Sarmiento A, Wagner $K$, Resnick C. The role of subtalar motion and ankle contact pressure changes from angular deformities of the tibia. Foot Ankle 1987; 7:290-9.

\title{
TOTAL KNEE REPLACEMENT FOR METASTATIC DESTRUCTION OF THE PROXIMAL TIBIA
}

\author{
R. M. TILLMAN, R. B. SMITH
}

Total joint replacement for metastatic disease of the hip is well established (Galasko 1986). Metastatic deposits in the proximal tibia requiring surgical intervention are rare, and we have found only one published report of prophylactic total knee replacement using a standard prosthesis for such a deposit (Beauchamp and Sim 1988). This procedure may present considerable technical challenges, but this case report demonstrates that, where successful, it can dramatically improve the quality of life for the terminally ill patient.

Case report. A 46-year-old man with advanced multiple myeloma had already undergone internal fixation for pathological fractures in all four limbs, but was now wheelchair bound and disabled by an extremely painful, large lytic metastasis in the right proximal tibia (Figs 1 and 2). There was imminent danger of fracture as only a cortical shell was left intact.

At operation packing with cement, bone grafting or internal fixation were not feasible due to the extent of the bone destruction. The collateral ligaments and the patellar tendon were, however, intact. Total knee replacement was therefore performed using a kinematic long stem 'stabiliser' prosthesis with a $21 \mathrm{~mm}$ tibial

R. M. Tillman, FRCS, Registrar

R. B. Smith, BSc, FRCS, Consultant

Department of Orthopaedic Surgery, Royal Preston Hospital, Sharoe

Green Lane, Preston, Lancashire PR2 4HT, England.

Correspondence to $\mathrm{Mr} \mathrm{R}$. M. Tillman.

(C) 1991 British Editorial Society of Bone and Joint Surgery 0301-620X/91/4R20 \$2.00

J Bone Joint Surg [Br] 1991 ; 73-B:516-7. component (Fig. 3). A triple mix of cement was used for the tibia and a plastic spatula was held at the back of the tibia to form a 'mould' where the posterior cortex had been completely destroyed, the spatula being removed when the cement had hardened. The alignment and stability were satisfactory (Fig. 4). It can be seen from the radiograph that the opportunity was taken to pack with cement a large distal femoral lesion previously fixed with a locking nail.

Postoperatively the patient was discharged home at eight days, pain free and able to walk. He was readmitted for terminal care nine months after the operation with no failure of the prosthesis.

Discussion. Metastatic lesions of the proximal tibia are relatively uncommon, accounting for only 36 of 399 longbone fractures in one large series (Harrington 1988). They typically present with pain and occasionally with fracture. Where fracture has occurred, open reduction and internal fixation with plate and screws preceded by curettage and possibly cement augmentation is required. In the absence of fracture, radiotherapy and external bracing may be appropriate, particularly where life expectancy is very short. Usually there is a vascularised lytic lesion surrounded by a shell of mainly intact but unsupported subchondral bone; the risk of fracture is high, increasing sharply when more than $50 \%$ of the cortex is involved (Fidler 1981). The weakened bone may be stabilised by curettage and packing with bone cement alone, or by rigid internal fixation. Rarely, amputation may be indicated for example, when a solitary metastasis is potentially curable. The major problem presented by reconstruction and arthroplasty is stability. Frequently 


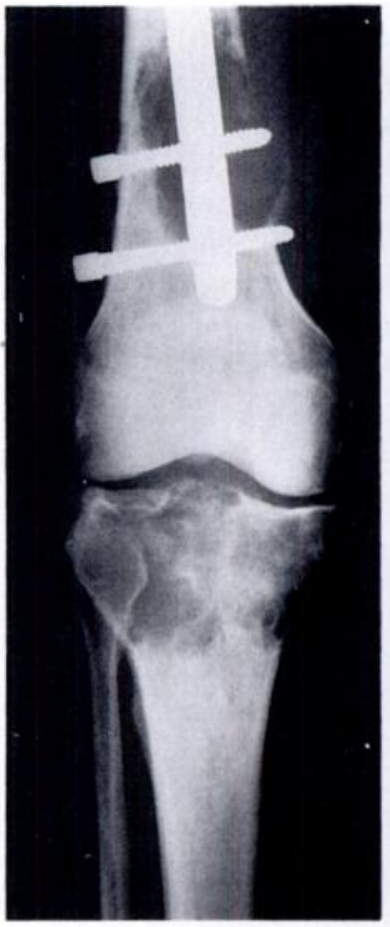

Fig. 1

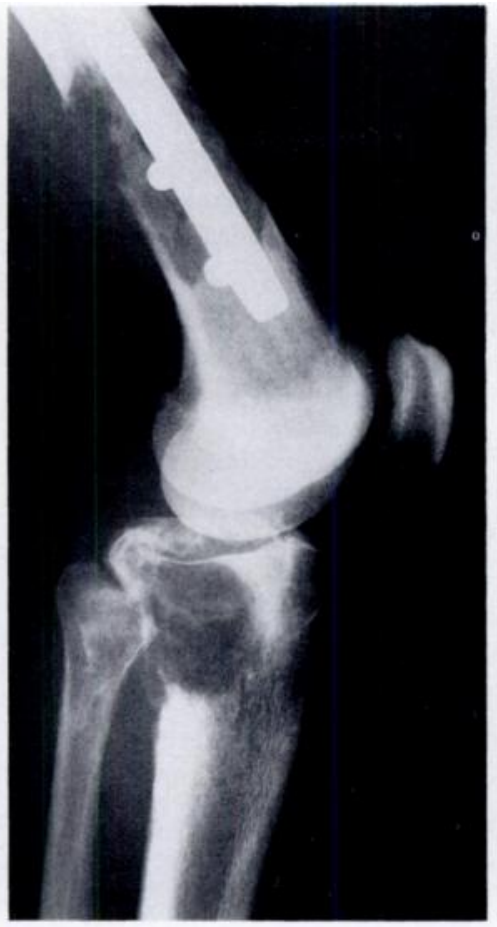

Fig. 2

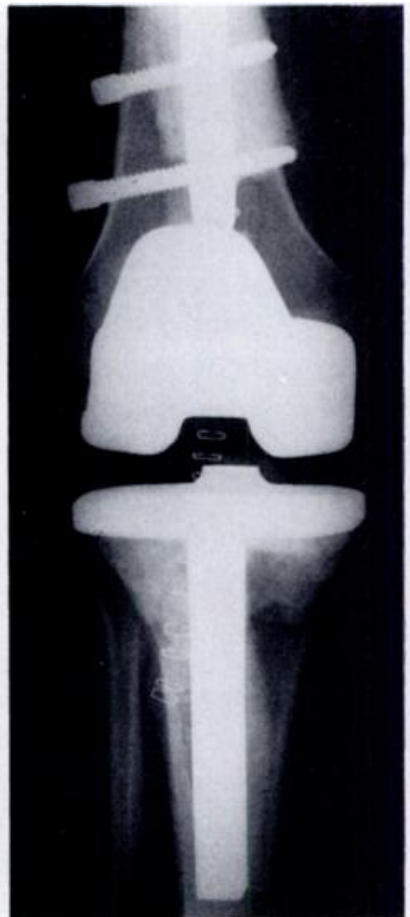

Fig. 3

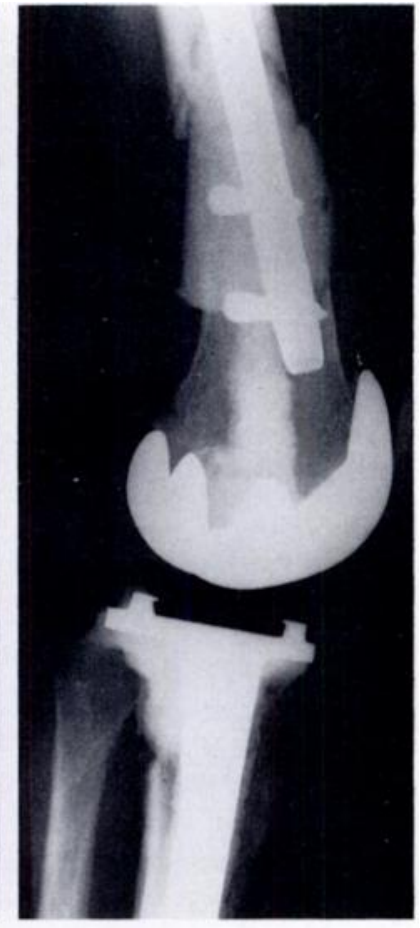

Fig. 4

the insertion of a collateral ligament or the patellar tendon has been destroyed and extensor function may be lost. A custom-made device is often necessary, and the delay involved may render this impractical in patients with a short life expectancy.

Operative treatment of skeletal metastases may have a high morbidity and must be aimed at improving the patient's quality of life. Our case illustrates that where survival even of a few months is likely, total knee arthroplasty can be an extremely rewarding and successful procedure.

No benefits in any form have been received or will be received from a commercial party related directly or indirectly to the subject of this article.

\section{REFERENCES}

Beauchamp CP, Sim FH. Lesions of the tibia. In : Sim FH, ed. Diagnosis and management of metastic bone disease. New York: Raven Press, $1988: 207-12$.

Fidler M. Incidence of fracture through metastases in long bones. Acta Orthop Scand 1981; 52:623-7.

Galasko CSB. Skeletal metastases. London: Butterworths, 1986:134-5.

Harrington KD. Orthopedic management of metastatic bone disease St Louis: CV Mosby, 1988.

\title{
AUTOLOGOUS BLOOD TRANSFUSION IN A DISTRICT HOSPITAL
}

\author{
A. GOEL, A. N. EDWARDS, N. C. WEST
}

An alternative to homologous blood transfusion is the use of the patient's own blood (autotransfusion) also known as autologous or predeposited blood transfusion (Kay 1987; Lee and Napier 1990). We have been using autotransfusion for over two years for hip and knee replacements; a brief review is presented.

A. Goel, FRCS, MS (Orth), Orthopaedic Registrar

A. N. Edwards, FRCS, Orthopaedic Consultant

N. C. West, MRCP, MRCP Path, Consultant Haematologist

West Cumberland Hospital, Whitehaven, Cumbria CA28 8JG, England.

Correspondence to Mr A. Goel.

(C) 1991 British Editorial Society of Bone and Joint Surgery $0301-620 X / 91 / 3 R 12 \$ 2.00$

J Bone Joint Surg [ Br] 1991; 73-B :517-8.
Methods. Our current protocol is that when the patient is put on the waiting list, he is given a fact sheet about autologous transfusion and the procedure is explained; if willing, a note is made in the records. Two weeks before the operation is due to take place the patient is admitted as a day case and reassessed from the point of view of surgery and autologous transfusion. If a decision is made to go ahead with the operation and if the patient is fit, a sample of blood is sent for haemoglobin estimation and blood screening.

The patient then goes to the haematology department where the results of the tests are recorded and, if the haemoglobin is adequate, one unit of blood is taken. He returns to the orthopaedic wards and after a brief period of observation is sent home with a prescription 